\begin{tabular}{ccc}
\hline & International Journal of Engineering \& Technology, $7(4.44)(2018) 194-197$ \\
SPC & International Journal of Engineering \& Technology \\
Website: www.sciencepubco.com/index.php/IJET & Research paper \\
\hline
\end{tabular}

\title{
Shallow Classifier with Sampling for Sleep Stage Classification of Autism Patients
}

\author{
Intan Nurma Yulita ${ }^{1 *}$, Mohamad Ivan Fanany, Aniati Murni Arymurthy \\ ${ }^{1}$ Universitas Indonesia \& Universitas Padjadjaran \\ ${ }^{2}$ Universitas Indonesia \\ ${ }^{3}$ Universitas Indonesia \\ *Corresponding author E-mail:intanurma@gmail.com
}

\begin{abstract}
Autism is a brain development disorder that affects the patient's ability to communicate and interact with others. Most people with autism get sleep disorders. But they have some difficulty to communicate, so this problem is getting worse. The alternative that can be done is to detect sleep disorders through polysomnography. One of the test purposes is to classify the sleep stages. The doctors need a long time to process it. This paper presents an automatic sleep stage classification. The classification was based on the shallow classifiers, namely naive Bayes, k-nearest neighbor (KNN), multi-layer perceptron (MLP), and C4.5 (a type of decision tree). On the other hand, this dataset has a class imbalance problem. As a solution, this study carried out the mechanism of resampling. The results show that the use of $d$ as a measure of the uniformity of data distribution greatly influenced the classification performance. The higher d, the more uniform the distribution of data $(0<=\mathrm{d}<=1)$. The performance with $\mathrm{d}=1$ was higher than $\mathrm{d}=0$. On the other hand, KNN was the best classifier. The highest accuracy and F-measure were 83.07 and 82.80 respectively.
\end{abstract}

Keywords: Classification, Sleep Stage, Autism, Imbalance Class, Machine Learning

\section{Introduction}

Children with autism have more difficulty sleeping than other children. In fact, the insomnia is experienced by 40-80 percent of children who have autism (Devnani \& Hegde, 2015). There are many factors that play a role in causing children with autism insomnia. The most common is the disruption of the production of the hormone melatonin which triggers sleepiness.

Normally, the melatonin levels increase at night and drop during the day (Hartzler, 2014). However, in children with autism, the opposite happens. The production of the hormone melatonin is influenced by certain amino acids in the body. In the children with autism, these amino acid levels are not balanced so the production of melatonin becomes higher during the day and drops dramatically at night. As a result, their sleep cycle is different from most children.

But children with autism have difficulty in conveying the problems that occur in sleep, including in terms of sleep disturbances. As a result, this condition causes their sleep problems to be more difficult to solve (Herrmann, 2016). The solution is to collect as much data as possible from people who know their bedtime habits. Another solution is identification through the application. This application is expected to recognize sleep disorders automatically through a number of data. The most widely used data is polysomnography (Haba-Rubio, 2012). It records brain waves, oxygen levels in the blood, heart rate and breathing, and eye and foot movements during the study. It is usually recorded in the sleep disorder units of the hospital or in the sleep laboratory. After the recording has been collected, then the doctors analyse the results.
One of which, they will identify or classify the sleep stage in the recording.

Some research related to the classification of sleep stages has been carried out (Aboalayon, Faezipour, Almuhammadi, \& Moslehpour, 2016). Langkvist et al implemented the hidden Markov model (Längkvist, Karlsson, \& Loutfi, 2014). The results obtained above $60 \%$ accuracy. Yulita et al were able to achieve accuracy above $70 \%$ through bi-directional long short term memory (Yulita, Fanany, \& Arymurthy, 2017a). Other studies that examine sleep stages apply a number of methods including support vector machine (Gouveris, 2017), random forest (Chung, Song, Cho, \& Chang, 2018), clustering (Fadhlullah, Resahya, Nugraha, \& Yulita, 2018), convolutional neural networks (Yulita, Fanany, \& Arymurthy, 2017b).

One of the main challenges in the sleep stage classification is a class imbalance problem. There are five stages of sleep according to the American Academy of Sleep Medicine (AASM). But the data proportion of each sleep stages has a different amount. If this problem is not addressed, then data will be classified into the most dominant class. This study proposes the use of sampling to handle it. It is also combined with the shallow classifier.

Moreover, until now, the research on sleep stage classification has been carried out on healthy respondents or sleep apnea (Boostani, Karimzadeh, \& Nami, 2017). But no study has discussed the sleep stage classification in autism patients. Therefore, another contributions of this study is the testing from autism patients sleep data. 


\section{Related study}

\subsection{Sleep stage}

Sleep is a natural human way to rest the body, renew damaged cells, and restore energy (Fullagar, et al., 2015). When sleeping, there is activity in the brain that goes through several stages called the sleep cycle. The sleep stage consists of two categories: rapid eye movement (REM), and non-REM (NREM). According to AASM, these two categories are divided into four stages (Berry, 2012), namely:

a) $\mathrm{N} 1$

Stage of light sleep as well as the transition stage between waking up and sleeping. If someone wakes up at this stage, he will feel he is not sleeping at all. In most people, it lasts 10 minutes.

b) $\mathrm{N} 2$

At this stage, eyeball movement stops, body temperature decreases, and heart rate begins to slow down. The body prepares to enter the deep sleep stage. It lasts for approximately 20 minutes.

c) $\mathrm{N} 3$

Is the stage of sleep in compared to the N2. At this stage there is absolutely no activity from the eyes and muscles in some parts of the body. This stage occurs 30 to 45 minutes from the time you first fall asleep.

d) $\mathrm{R}$

This stage is characterized by extensive blood pressure, pulse and breathing frequency. It is a period of sleep characterized by eye movements, loss of muscle strength and dreams that appear real (Lucid Dream). It is also called high brain activity in the body that is paralyzed or paradoxical. At this moment, clear dreams appear more often.

The non-REM cycle (NREM) consists of stages N1, N2, and N3. This cycle lasts for approximately 90-120 minutes. After being in stage N3, the sleep cycle will repeat to $\mathrm{N} 2$ before finally entering the stage of REM. REM is represented by stage R. The normal sleep cycles generally follow the following pattern: the waking conditions, stages N1, N2, N3, N2, REM. A person usually experiences five cycles of sleep when resting at night.

\subsection{Sampling}

The accuracy of the data class is very important in machine learning classification. The more accurate the data set and class is, the better the output produced by machine learning. In reality the classification can experience an imbalance class. The imbalance class is an imbalance in the amount of training data between two different classes, one of the classes represents a very large amount of data (majority class) while the other class represents a very small amount (minority class). The imbalanced data problem can cause the results of calcification to be inaccurate. A technique is needed to make it balanced so that it can produce accurate prediction (Estabrooks, 2004).

By increasing minority classes, it can improve the ability of the technology learning algorithm to be better. It can recognize minority class samples from the majority sample. Resampling is the most popular way to overcome this problem. There are three basic approaches to overcome the problem of class imbalance, namely oversampling of the minority-class, under-sampling of the majority class or using a hybrid method that uses the basis of these two methods. It is also a means of changing the distribution of minority classes so that they are not underrepresented when training data on mechanics learning algorithms.

The resampling method is well-known to be applied to avoid imbalance classes. Over-sampling is the simplest method for dealing with minority classes by conducting random classes during the sampling process. The sampling process with this oversampling technique is to duplicate the positive class and do random class balancing. However, because this method duplicates the positive class that is in the minority class, the possibility of over-fitting will definitely occur. Under-sampling is almost the same as oversampling technique by calculating the difference between the majority class and the minority class. Then repeated as many as the majority class difference with the minority-class. During the repetition process, the majority class is eliminated so that the same number is obtained with the minority class.

\subsection{Shallow classifier}

Machine learning brings humans and machines together with the human approach to "learn" machines (Fernández-Varela, Hernández-Pereira, vlvarez-Estévez, \& \& Moret-Bonillo, 2017). The machines can learn by processing exercises that contain important features to adjust to algorithms through large data. One of the goals of machine learning is classifications (Yulita, Fanany, \& Arymurthy, 2018). There are two approaches to classification: shallow and deep learning. In this study, the classification based on shallow learning. Four shallow classifiers were used in this study, namely naive Bayes, k-nearest neighbor (KNN), multi-layer perceptron (MLP), and C4.5 (a type of decision tree).

\subsubsection{Naive Bayes}

Naïve Bayes is a statistical grouping that can be used to predict the probability of members of a class (Zhou, 2015). It also has very strong accuracy and speed when applied to databases with big data The method uses conditional probabilities as the basis. In statistics, the conditional probability is stated as Eq.1.

$P(X \mid Y)=\frac{P(X)}{P(Y)} P(Y \mid X)$

\subsubsection{K-nearest neighbor (KNN)}

K-nearest neighbor is an approach to look for cases by calculating the closeness between new cases and the old case, which is based on matching weights of a number of features (Tian, 2016). It is desirable to look for the target class of a segment by using the target class from known segments. The prediction is based on the greatest closeness that will be taken to be used in the segment. As for one way to measure the distance of the data proximity of a sample with other data can use the Euclidean distance.

\subsubsection{Multi-layer perceptron (MLP)}

Artificial neural networks (ANN) are inspired by the structure and function of the human brain. In an ANN there are a number of neurons (Mirjalili, 2014). One neuron can connect for many other neurons, and each connection has a weight. A learning is a basic characteristic of biological neural networks. The ANN conducts the learning process through adjusting the weight on their connections. Multilayer perceptron is the most common topology of ANN, where the perceptron are connected to form several layers. An MLP has an input layer, at least one hidden layer, and an outer layer. The most widely used method in MLP learning is backpropagation. There are four steps that must be done in this method, namely initialization, activation, weight training, and iteration. In the initialization step, the initial weight values and thresholds are determined randomly but within certain limits. At the activation stage, the input and expected output values are given. The process of weight adjustment occurs at the weight training stage, the actual output value is compared with desired output and weight adjustments are made. The second and third steps are repeated until a specified condition is reached. 


\subsubsection{C4.5}

C4.5 algorithm is a decision tree algorithm group. This algorithm has input in the form of training samples and samples. Training samples are sample data that will be used to build a tree that has been tested properly. While samples are data fields which we will use as parameters in classifying data (Lajnef, 2015). The algorithm is one method for making a decision tree based on the training data that has been provided. The algorithm is the development of ID3. Some of the developments carried out on C 4.5 are as follows, among others, can overcome missing value, and continued data.

In general, the $\mathrm{C} 4.5$ algorithm for building decision trees is as follows:

a) Select the attribute as root

b) Create a branch for each value

c) Share cases in branches

d) Repeat the process for each branch until all the cases in the branch have the same class.

To choose an attribute as root, it is based on the highest gain value of the existing attributes. Before getting the gain value is to find the entropy value. The entropy is used to determine how informative an input attribute is to produce an attribute.

\section{System design}

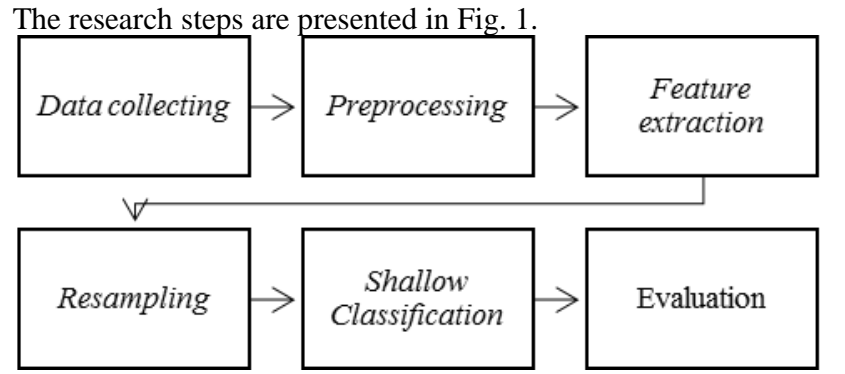

Fig. 1: Flow chart that represents the research steps for this sleep stage classification

\subsection{Data collecting}

The recording was done in Mitra Keluarga Kemayoran hospital, Jakarta that provides the polysomonography. The implementation was carried out at the end of 2017. Thirteen autism patients were involved, but data from 7 people could be used in this study. The constraints during collection were difficult to find patients who were willing to be involved in this recording. In addition, some patients experienced tantrums during the recording process so the recording was not successful. After recording was done, the doctor analyzed the types of sleep stages per 30 seconds. The scoring rules based on the American academy of sleep medicine (AASM). A number of electrodes were installed from head to toe. It recorded 32 signals. Fig. 2 is one example of our polysomonography from an autism patient. The figure consists of several signals originating from EEG. The signals shown are channels from M1, C3, M2, and C4. But this study only used a channel from EEG. We chose the $\mathrm{C} 4$ channel because many studies show that its performance is best for the sleep stage classification.

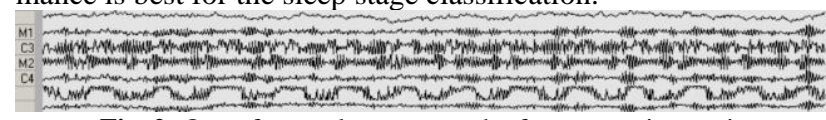

Fig. 2: One of our polysomnography from an autism patient

\subsection{Pre-processing}

At this stage, the signal was filtered. This study applied notch and band pass filtering. Notch filters reject frequencies that will not be used at a later stage. This study removed a $50 \mathrm{~Hz}$ tone from a signal in its sample rate. The band-pass filter passed signals with frequencies in a certain range and reduced the signal with other frequencies. It was filtered with a band-pass filter of 0.3 to the half of its sample rate size. Also the signal was down sampled to the half of its sample rate size.

\subsection{Feature extraction}

17 features were extracted from a signal of $\mathrm{C} 4$. These features were obtained from dual tree complex wavelet transform with two levels, fast Fourier transform, fractal exponent, kurtosis, entropy, and spectral mean.

\subsection{Resampling}

The resampling was done by taking random data from the initial dataset. After being selected, the data was returned again. It allowed the same data to be re-elected. This study conducted three experiments based on the number of class distributions. If the class distribution (d) was 0 then it ignored the taken sleep stage. But if the class distribution (d) was 1, then the new dataset had a uniform number of class distributions.

\subsection{Shallow classification}

Four types of shallow classifier were used in this study: naïve Bayes, KNN, MLP, and C4.5. The KNN was tested with $\mathrm{K}$ values from 1 to 5 . The number of hidden layers of MLP was 11, and the learning rate was 0.3 .

\subsection{Evaluation}

The four types of evaluation parameters used in this study were accuracy, precision, recall, and f-measure. The accuracy is the level of proximity of the quantity measurement to the actual value. Precision is the level of accuracy between the information requested and the answer given by the system. Whereas recall is the success rate of the system in rediscovering information. F-measure is a combination of precision and recall.

\section{Results}

The experiment was conducted by testing the four algorithms namely naïve Bayes, KNN, MLP, and C4.5. The four algorithms were tested on two experiments with changes in the value of the distribution class variable (d). The results are shown in Table 1, 2.

Table 1: Results from each algorithm with $\mathrm{d}=0$

\begin{tabular}{|c|c|c|c|c|}
\hline \multirow{2}{*}{ Method } & \multicolumn{4}{|c|}{$\%$} \\
\cline { 2 - 5 } & Accuracy & Precision & Recall & F-measure \\
\hline Naïve Bayes & 34.19 & 46.60 & 34.20 & 34.50 \\
\hline KNN (K=1) & 81.60 & 81.60 & 81.60 & 81.60 \\
\hline KNN (K=2) & 71.05 & 71.00 & 71.00 & 70.80 \\
\hline KNN (K=3) & 68.03 & 67.80 & 68.00 & 67.70 \\
\hline KNN (K=4) & 68.01 & 67.60 & 68.00 & 67.30 \\
\hline KNN (K=5) & 67.47 & 67.00 & 67.50 & 66.90 \\
\hline MLP & 62.13 & 60.30 & 62.10 & 57.80 \\
\hline C4.5 & 79.64 & 79.40 & 79.60 & 79.50 \\
\hline
\end{tabular}

Table 1 shows that the performance of all classifiers with $\mathrm{d}=0$. Naiive Bayes have the worst performance, while KNN is the best. The lowest and highest accuracy were $34.19 \%$ and $81.60 \%$, respectively. If the $\mathrm{K}$ value of KNN got bigger, the performance of the KNN gets worse. The variations between data in the same class were quite high. The more neighbors that were used, the data captured is data that has a different class. Also, this experiment showed MLP and C.45 also had an accuracy of $62.13 \%$ and $79.64 \%$, respectively.

Table 2 shows the performance of the four algorithms with $d=1$. Almost all classifier showed the improved performance. The accuracy of $\mathrm{KNN}$ was the highest with $\mathrm{K}=1$. It was $83.07 \%$. But if 
more than two neighbors of KNN, the performance decreased. In this experiment, the MLP performance decreased. Other methods include naïve Bayes got their highest performance with $\mathrm{d}=1$. It can be concluded that the best class distribution was 1 . It means the balanced classes was needed to overcome the problems in this case study.

Table 2: Results from each algorithm with $\mathrm{d}=1$

\begin{tabular}{|c|c|c|c|c|}
\hline \multirow{2}{*}{ Method } & \multicolumn{4}{|c|}{$\%$} \\
\cline { 2 - 5 } & Accuracy & Precision & Recall & F-measure \\
\hline Naïve Bayes & 36.88 & 36.70 & 36.90 & 34.90 \\
\hline KNN (K=1) & 83.07 & 83.10 & 83.10 & 82.80 \\
\hline KNN (K=2) & 74.20 & 73.60 & 74.20 & 73.80 \\
\hline KNN (K=3) & 70.94 & 70.00 & 70.90 & 70.10 \\
\hline KNN (K=4) & 70.56 & 69.60 & 70.60 & 69.60 \\
\hline KNN (K=5) & 69.40 & 68.60 & 69.40 & 68.20 \\
\hline MLP & 52.36 & 53.40 & 52.40 & 52.20 \\
\hline C4.5 & 81.81 & 81.20 & 81.80 & 81.40 \\
\hline
\end{tabular}

\section{Conclusion}

With $\mathrm{d}=0, \mathrm{KNN}$ with $\mathrm{K}=1$ was the best performance in this study, as shown in Table 1 . The accuracy of KNN reached $83.07 \%$. Naive Bayes had the worst performance. . Also, this experiment showed MLP and C.45 also had an accuracy of $62.13 \%$ and $79.64 \%$, respectively. If $\mathrm{d}=1$, all classifier had the improved performance, except MLP. The MLP performance was higher $\mathrm{d}=0$ than $d=1$. This indicates that resampling as a solution of imbalance class problems was very necessary. If a class dominated, the system tend to choose the dominant class. It caused the accuracy to be small because the data with minor classes could not be correctly classified.

\section{Acknowledgement}

This work supported by Center of Excellence for Higher Education Research Grant funded by Indonesian Ministry of Research and Higher Education. This paper also backed by GPU grant from NVIDIA.

\section{References}

[1] Aboalayon, K., Faezipour, M., Almuhammadi, W., \& Moslehpour, S. (2016). Sleep stage classification using EEG signal analysis: a comprehensive survey and new investigation. Entropy, 272.

[2] Berry, R. B. (2012 ). Rules for scoring respiratory events in sleep: update of the 2007 AASM manual for the scoring of sleep and associated events. Journal of clinical sleep medicine 8(05), 597-619.

[3] Boostani, R., Karimzadeh, F., \& Nami, M. (2017). A comparative review on sleep stage classification methods in patients and healthy individuals. Computer methods and programs in biomedicine, $77-$ 91.

[4] Chung, K., Song, K., Cho, S., \& Chang, J. (2018). Noncontact Sleep Study Based on an Ensemble of Deep Neural Network and Random Forests. IEEE Sensors Journal.

[5] Devnani, P., \& Hegde, A. (2015). Autism and sleep disorders. Journal of pediatric neurosciences 10(4).

[6] Estabrooks, A. J. (2004). A multiple resampling method for learning from imbalanced data sets. Computational intelligence, 20(1), 18-36.

[7] Fadhlullah, M., Resahya, A., Nugraha, D., \& Yulita, I. (2018). Sleep stages identification in patients with sleep disorder using kmeans clustering. Journal of Physics: Conference Series , 1013(1), $1-5$.

[8] Fernández-Varela, I., Hernández-Pereira, E., Álvarez-Estévez, D., \& \& Moret-Bonillo, V. (2017). Combining machine learning models for the automatic detection of EEG arousals. Neurocomputing (268), 100-108.

[9] Fullagar, H., Skorski, S., Duffield, R., Hammes, D., Coutts, A., \& Meyer, T. (2015). Sleep and athletic performance: the effects of sleep loss on exercise performance, and physiological and cognitive responses to exercise. Sports Medicine , 161-186.
[10] Gouveris, H. e. (2017). Sleep stage classification using spectral analyses and support vector machine algorithm on C3-and C4-EEG signals. Sleep Medicine, 40, e116.

[11] Haba-Rubio, J. \&. (2012). Evaluation instruments for sleep disorders: A brief history of polysomnography and sleep medicine. Introduction to modern sleep technology, 19-31.

[12] Hartzler, B. (2014). Fatigue on the flight deck: the consequences of sleep loss and the benefits of nappin. Accident Analysis \& Prevention, 309-318.

[13] Herrmann, S. (2016). Counting sheep: sleep disorders in children with autism spectrum disorders. Journal of Pediatric Health Care, 143-154.

[14] Lajnef, T. e. (2015). Learning machines and sleeping brains: automatic sleep stage classification using decision-tree multi-class support vector machines. Journal of neuroscience methods , 250, 94-105.

[15] Längkvist, M., Karlsson, L., \& Loutfi, A. (2014). A review of unsupervised feature learning and deep learning for time-series modeling. Pattern Recognition Letters, 11-24.

[16] Mirjalili, S. M. (2014). Let a biogeography-based optimizer train your multi-layer perceptron. Information Sciences, 269, 188-209.

[17] Tian, J. M. (2016). Motor bearing fault detection using spectral kurtosis-based feature extraction coupled with K-nearest neighbor distance analysis. IEEE Transactions on Industrial Electronics 63(3), 1793-1803.

[18] Yulita, I. N., Fanany, M., \& Arymurthy, A. (2018). Fast Convolutional Method for Automatic Sleep Stage Classification. Healthcare informatics research, 24(3), 170-178.

[19] Yulita, I., Fanany, M., \& Arymurthy, A. (2017a). Bi-directional Long Short-Term Memory using Quantized data of Deep Belief Networks for Sleep Stage Classification. ICCSCI (pp. 530-538). Bali: Procedia Computer Science.

[20] Yulita, I., Fanany, M., \& Arymurthy, A. (2017b). Sleep stage classification using convolutional neural networks and bidirectional long short-term memory. 2017 International Conference on Advanced Computer Science and Information Systems (ICACSIS) (pp. 303-308). Jakarta: IEEE.

[21] Zhou, X. W. (2015). etection of pathological brain in MRI scanning based on wavelet-entropy and naive Bayes classifier. International conference on bioinformatics and biomedical engineering (pp. 201209). Springer. 